\title{
Design and Development of Mobile Games Based on Android Platform
}

\author{
Junpeng He \\ Xi’an International University, Shaanxi Xi’an 710077
}

Keywords: Android platform; mobile games; design and development

\begin{abstract}
With the popularization of smart phones, more and more users are willing to enjoy the pleasure of playing on mobile phones. Therefore, many game companies will regard mobile phone games as the key of development. At present, the main platform for mobile games is IOS platform, Android platform, and Mobile platform. This paper is made on the basic of Android platform for mobile game design and development. After the analysis of the current needs of mobile games, the design and development were carried out.
\end{abstract}

\section{Introduction}

More and gaming companies have good prospects for development of mobile games. Related research shows that users will choose to download mobile games when downloading applications accounting for about $40 \%$ [1]. Data shows that mobile games have a large user base [2]. With the current smart phone, most of the mobile phone games support network connection. From the current mobile phone game development status and development trends, it can already be comparable with handled games. With the technical support of modern mobile games, they are no longer the original screen rough and single game, but a strong entertainment and interactive game software. From the current status, mobile games have become the most rapid growth of mobile multimedia services business activities [3]. If you want to design a mobile phone game, the first thing you need to do is to understand their needs.

\section{Game needs}

Now telephone is not a simple communication tool. With the continuous development of the mobile game industry, mobile game development has a variety of mature implementation methods and technical means. However, you want to design and develop a public favourite game, you need to stand in the user's point of view to give users the best gaming experience.

(1) Analysis of a general process of the game: the user open the game $\rightarrow$ select the interface to view $\rightarrow$ the operation on the interface $\rightarrow$ feedback prompt information. Inside the game operation phase: start the game $\rightarrow$ game screen preparation $\rightarrow$ game character operation $\rightarrow$ game score prompts.

(2) Analysis of the functional requirements of the game: including game display, game operation, game scene conversion, game sound settings, and game data management. The game shown in Figure 1.

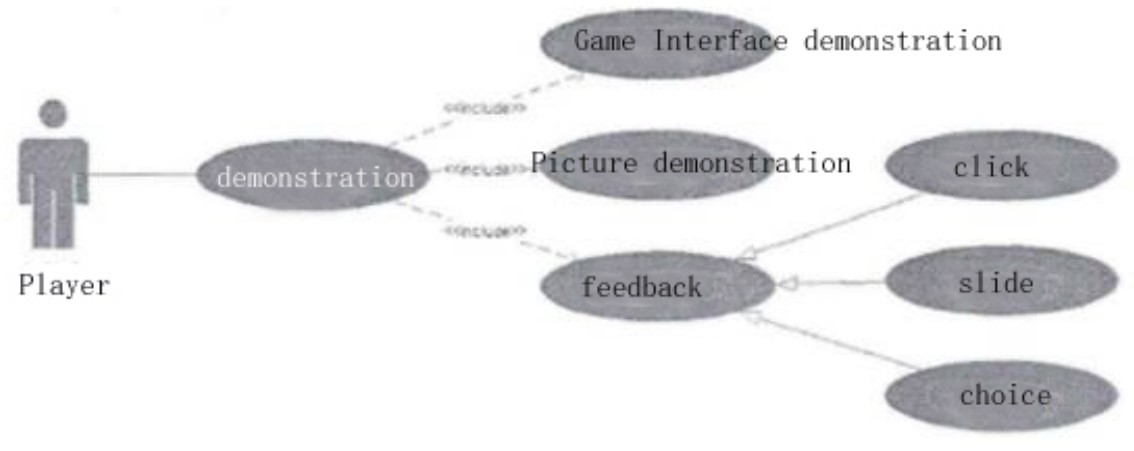

Figure 1 shows a game use case diagram 
(3) Non-functional requirements of the game: non-functional requirements analysis of performance requirements and security needs. Performance requirements are divided into maintainability, ease of operation, scalability, openness. Safety requirements are divided into fault tolerance and operational safety.

\section{Game Design Based on Android Platform}

The design of the game is divided into five large modules, including the game front rendering engine, game control engine, game logic module, game sound module and game data persistence module. Different modules are responsible for different directions [4]. We take game control engine as an example. Its implementation is different on the game interface management and switch between the data communication components. There is a specific analysis of each module design followed.

(1) Game foreground rendering engine module: The module's main function is to display the game interface, game screen and game character animation. Another very important function is responsible for the game control engine and game logic module provides game interface. The game interface also includes button clicks, list options, and gesture swipes. The core part of the module processing are as following: access to interface operation information $\rightarrow$ button click processing, click list option processing, gesture slide processing $\rightarrow$ game control engine processing.

(2) Game control engine module: The module's main function is to coordinate the good operation between each module, also includes the communication between components and game interface switching management. Game control engine is divided into game interface switching control (click the interface button, click the keyboard button) and the game component data communication (information carrier, send broadcast messages, receive broadcast information).

(3) Game logic module: through Android gravity sensing technology to achieve the state of the game screen control. As the gravity sensor parameter changes will affect the state of the game screen [5]. So the game logic module analyses gravity sensing, game screen rendering and cell phone vibrators. The game screen is divided into picture state switching and character animation rendering. Character Animation Class diagram: -canvas

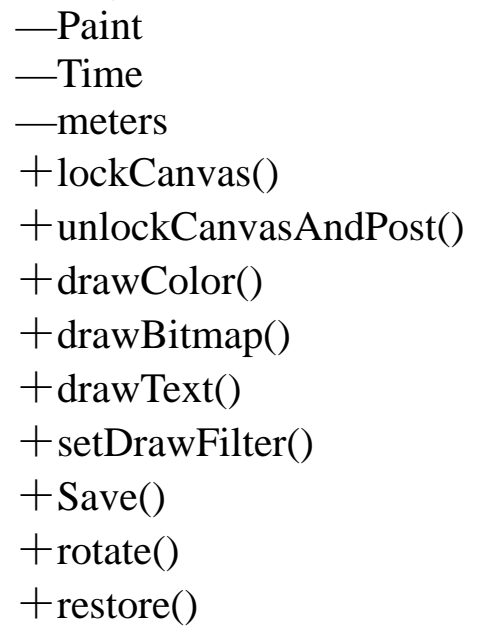

The game screen state conversion process: ready to state the game screen processing $\rightarrow$ live game screen processing $\rightarrow$ (click on the start icon) $\rightarrow$ effective state of the game screen processing $\rightarrow$ moving screen playback $\rightarrow$ left wrestling animation, right wrestling animation $\rightarrow$ dead state game screen processing. Gravity sensing motion: Obtains the screen length and width and calculates the screen coordinates of the game screen control points. $\rightarrow$ Gravity sensing monitors the rotation offset of the $\mathrm{x}, \mathrm{y}$ and $\mathrm{z}$ axes. $\rightarrow$ Changes the game screen control according to the $\mathrm{x}, \mathrm{y}$ axis rotation offset. Point screen coordinates, the screen coordinates of the screen changes to determine the state, according to the z-axis rotation of the size of the amount of deviation in the game screen rendering warm tips $\rightarrow$ whether to end monitoring.

(4) Game music module: the module provides sound support, the main function is to set the 
media volume, play the game background music and game effects music. Music playback class diagram:

$$
\begin{aligned}
& \text { + start() } \\
& \text { + stop() } \\
& \text { + play() } \\
& \text { Volume settings: } \\
& \text { + setVolumeControIStreem } \\
& \text { + getStreamMaxVolume() } \\
& \text { + getStreamVolume() } \\
& \text { + getStreamVolume() }
\end{aligned}
$$

(5) Game data persistence module: including player information storage, game option information storage (file access), game ranking information storage (data adapter, database operation).

(6) On the database design, including the game ranking information table, see Table 1.

Table 1 game ranking information table

\begin{tabular}{|l|l|l|l|l|l|l|}
\hline name & type & length & $\begin{array}{l}\text { Allow } \\
\text { empty }\end{array}$ & $\begin{array}{l}\text { Primary } \\
\text { key }\end{array}$ & $\begin{array}{l}\text { Foreign } \\
\text { key }\end{array}$ & Remarks \\
\hline ID & Varchar & 32 & no & yes & no & \\
\hline Scores & Varchar & 4 & no & no & no & \\
\hline Date & Varchar & 8 & no & no & no & \\
\hline -Time & Varchar & 8 & no & no & no & \\
\hline UserNnme & Varchar & 20 & no & no & no & \\
\hline
\end{tabular}

\section{Game Design Based on Android Platform}

Game development environment is the based on Android mobile game development. This study used Huawei mate8 real machine test. Eclipse and Eclipse integration in the Andrews ADT and Andrews 2.1SDK components, the installation of Huawei mate8 mobile phone driver, the use of jdk1.6.0_20 and configure the Java system environment variables. Complete the phone's debugging work. It began to enter the creative stage.

(1) Game interface production: the use of Adobe Photoshop software for graphics and image processing. On the role of the game animation, including standing animation, the role of walking animation, the role of the left wrestling animation and the role of right-wrestling animation. One of the characters standing animation needs to loop, so the first frame and the last frame must overlap. The character animation pictures are in PNG format, and all the characters of the game characters are processed by Adobe Photoshop.

(2) On the game front rendering engine: rendering the game screen, the only rendering active, active, dead state of the game screen. On the game interface, in this game design, monitor the click interface in the game button is onTouchEvent (), you can consider this method by clicking on the button to achieve the judgement. In addition, the game's help interface can use the left slide gesture and the right slide gesture to view the picture.

(3) On the game control engine: the engine as a core part of the game [6], its purpose is to coordinate the operation of each module to ensure that the communication between the various components, the game interface management switch. For the data communication between components, through the use of Activity and Service between the completion of communications, which are based on Intent and Broadcast Receiver completed. The key code:

Intent intent=new Intent (activity, GgmeService, class);

activity.startService (intent);

The code is a background thread that launches music for playing background music and game effects. 
(4) On the game interface switch management: a game can not only have a game interface, so need to do the game interface management. A game interface corresponds to a game interface resource ID. Game interface resource ID is generated by the interface button and the operation of the keyboard button.

(5) On the game logic module, which includes the game screen rendering, gravity sensing, mobile phone vibrator system, the game canvas rotation angle calculation, part of the game character animation area calculation. To gravity sensing, for example, the use of Andrews gravity sensing technology, in which the gravity sensing parameter values through the game screen control point to receive. Game screen control point determines the state of the game screen and the game character animation [7], part of the key code:

zPosition=sensorEvent.values $\{2\}$; //Phone screen facing

//zPosition $>0$ the phone screen is facing up //zPosition $<0$ Phone screen facing

(6) Game audio module and game data persistence module: game audio module, including Audio Manger adjust the media volume, MediaPlayer play game background music, SoundPool play game special effects music. In this paper, we use SoundPool to play the game special effects music, for example, we design the different game effects for the different characters. When the player starts the game, all the game background thread has been loaded, different game screen state trigger different game effects music. As the game will be involved in a lot of game data, a lot of information needs the game data persistence module to achieve access.

\section{Conclusion}

Andrews phone with the popularity of more and more advanced technology, developed for the Andrews platform for mobile games more and more attractive screen, more and more content, creativity more and more obvious. From the real needs of mobile games, this research analyzes the three aspects of demand, design and development, hoping to provide reference for related design and R \& D work.

\section{References}

[1] Based on the Andrews platform for the design of mobile phone games puzzle [J]. Li Yang. "Journal of Huaihai Institute of Technology (Humanities and Social Sciences), 33 (16): 88-90, 2012.

[2] Design and implementation of J2ME mobile game development platform [J]. Yuehua Ding, Jia iu, Pei Yang. Computer Engineering, 29 (05): 261-263, 2007.

[3] Based on the Andrews platform puzzle game development [J]. Xiaodan Zhao. 53 (49): 222, 2015.

[4] The design and implementation of the game "extremely connected" based on Android platform [J]. Mingming Wu, Xin Zhang, Wenqiang Dong, Shuai Shao, Fanyu Liu. Modern Industrial Economy and Informatization, 33 (09): 104-105 + 112, 2016.

[5] Application of Android platform on the design and development of handheld campus APP [J]. Yicong Zhang. Information and Computer (Theoretical Edition), 37 (07): 87-88 2016.

[6] Based on the Android system design and development of mobile games [J]. Zhenghui Pan. Electronic World, 26 (13): $128+$ 130, 2016.

[7] Design of Sudoku Game Based on Android Platform [J]. Yang Wang, Zhiguang Chu. Journal of Liaoning University of Technology (Natural Science Edition), 29 (04): 242-245, 2013. 